\title{
Original
}

\section{Biological Control of Xanthomonas Oryzae pv. Oryzae Causing Rice Bacterial Blight Disease by Streptomyces toxytricini VN08-A-12, Isolated from Soil and Leaf-litter Samples in Vietnam}

\author{
DUONG VAN HOP ${ }^{1}$, PHAN THI PHUONG HOA ${ }^{1,4 *}$, NGUYEN DUC QUANG ${ }^{2}$, \\ PHAN HUU TON ${ }^{3}$, TRINH HOANG HA ${ }^{4}$, NGUYEN VAN HUNG ${ }^{3}$, NGUYEN THI VAN ${ }^{1}$, \\ TONG VAN HAI ${ }^{3}$, NGUYEN THI KIM QUY', NGUYEN THI ANH DAO ${ }^{1}$, AND VU THI THOM ${ }^{4}$ \\ ${ }^{1}$ Institute of Microbiology and Biotechnology (IMBT), Vietnam National University, Hanoi (VNUH) \\ E2 Building, 144 Xuan Thuy road, Cau Giay District, Hanoi Province, Vietnam \\ ${ }^{2}$ University of Engineering and Technology, Vietnam National University, Hanoi \\ ${ }^{3}$ Faculty of Biotechnology, Hanoi University of Agriculture \\ ${ }^{4}$ School of Medicine and Pharmacy, Vietnam National University, Hanoi
}

Received 13 March, 2013/Accepted 13 December, 2013

\begin{abstract}
A total of 2690 actinomycete strains were screened as potential biological control agents in controlling rice bacterial blight (BB) in Vietnam. From these microorganisms, seventeen actinomycete strains were found to be capable of inhibiting all 10 major Xoo races isolated from Xoo-infected rice leaves. One strain, namely VN08-A-12, contained effective characteristics in selectively inhibiting all 10 Xoo races in vitro, but did not inhibit most of the other tested microorganisms. Therefore, VN08-A-12 was subsequently selected for rice field trials for two seasons on two rice cultivars SS1 and KD18. Results showed VN08-A-12 was not only able to reduce Xoo lesion lengths in the two rice cultivars (lesion length reduction of up to $38.3 \%$ ), but it also significantly reduced Xoo-related yield loss in infected rice cultivars from the field (yield loss reduction of up to $43.2 \%$ ). Interestingly, the culture of this strain also increased the rice yield in healthy rice cultivars (from $2.66 \%$ to $16.98 \%$ for SS1 and from $3.11 \%$ to $5.94 \%$ for KD18 cultivar). The strain VN08-A-12 was shown to be identical to Streptomyces toxytricini. To our knowledge, this is the first study reporting $S$. toxytricini as a beneficial biological agent for the control of BB in rice.
\end{abstract}

Key words : Xoo / Streptomyces toxytricini / Vietnam.

\section{INTRODUCTION}

Bacterial blight (BB) of rice is caused by Xanthomonas oryzae pv. oryzae (Xoo), and is one of the most destructive diseases of this essential crop. The disease can lead to yield losses of up to 50 or 60 $\%$ in tropical regions throughout Asia (Adhikari et al., 1994; Dinh et al., 2008; Gnanamanickam, 2009) and it has occurred in many rice cultivating areas of Vietnam (Dinh et al., 2008). In addition to using chemicals and

*Corresponding author. Tel and Fax: +84-437547407, E-mail : phuonghoa(a) gmail.com varietal resistance, researchers have recently attempted to control BB in rice by using biological agents and their bioactive compounds (Chithrashree et al., 2011; Ndonde and Semu, 2000). The use of microorganisms for controlling $X_{O O}$ has shown significant promise in being an ecologically-conscious and cost-effective solution in rice cultivation (Gnanamanickam, 2009; Park et al., 2011).

Actinomycetes are gram-positive bacteria that are well-known as a source of natural products (Anzai et al., 2008). It has been reported that of the approximately 10,000 antibiotics discovered from microorganisms, two-thirds were isolated from actinomycetes 
(Okami and Hotta, 1988). Actinomycetes, mainly the genus Streptomyces, have produced a wide variety of bioactive compounds, including antibiotics (Anzai et al., 2008). In recent studies, Streptomyces activity against Xoo strains has been reported (Ndonde and Semu, 2000; Yan et al., 2000; Park et al., 2011) and some bioactive compounds have been identified (Park et al., 2011), suggesting that actinomycetes are potential biological agents for the control of XoO.

In the present study, from a collection of 2690 actinomycete strains, which had been isolated from 2005 to 2010 and preserved at the Vietnam Type Culture Collection (Institute of Microbiology and Biotechnology, Vietnam National University Hanoi), we screened for actinomycete strains that would be able to inhibit all 10 major Xoo races occurring in northern Vietnam and Japan (Ton et al., 2003; Hoa et al., 2012). An actinomycete strain named VN08-A-12 was selected as a possible biological control agent of BB. This strain was selected for its potential to inhibit $X_{O O}$ races, reduce Xoo lesion lengths in rice leaves and reduce $X_{0 o}$-related yield losses in infected rice cultivars from field trials. The strain was identified to the species level.

\section{MATERIALS AND METHODS}

\section{Experimental design}

A collection of 2690 actinomycete strains was used to test for inhibitory actions against Escherichia coli or Micrococcus luteus. 167 strains that had high inhibition activity against $E$. coli or $M$. luteus were selected. We then screened for the strains that inhibited all 10 Xoo races among those 167 strains. 17 strains that inhibited all 10 Xoo races were selected. Then, the antagonistic tests against indicator strains [M. luteus NBRC 13867, E. coli NBRC 14237, Bacillus subtilis NBRC 3134 and Saccharomyces cerevisiae NBRC 10217] and useful microogarnisms [Azotobacter sp. (VTCC-B-106) and Pseudomonas putida (VTCC-B-657)] were done for the 17 selected strains. The strain named VN08-A12 was selected as a potential strain for rice field trials over two cropping seasons; then we identified this strain to the species level.

\section{Actinomycete strains}

During 2005 to 2010 at the Vietnam Type Culture Collection (Institute of Microbiology and Biotechnology, Vietnam National University Hanoi), over 3000 actinomycete strains were isolated from soil and leaf-litter samples (Hop et al., 2011; Hoa et al., 2012) by using four methods in conjunction with humic acid-vitamin agar as an isolation medium: the rehydration-centrifugation (RC) method, sodium dodecyl sulfate-yeast extract dilution method, dry-heating method and the
TABLE 1. Collection of actinomycete strains from 2005 to 2010

\begin{tabular}{cccc}
\hline $\begin{array}{c}\text { Name of } \\
\text { strains }\end{array}$ & $\begin{array}{c}\text { Year of } \\
\text { isolation }\end{array}$ & $\begin{array}{c}\text { Obtained and } \\
\text { tested strains }\end{array}$ & References \\
\hline VTCC & 2005 & 600 & \\
VN07 & 2007 & 1000 & Hop et al., 2011 \\
VN08 & 2008 & 500 & \\
VN10 & 2010 & 90 & Hoa et al., 2012 \\
Total & & 2690 & \\
\hline
\end{tabular}

oil-separation method (Hop et al., 2011). Among these strains, a total of 2690 actinomycete strains were used to screen for antibiotic-producers by assay for antagonism to M. luteus NBRC 13867 and E. coli NBRC 14237. The method was previously mentioned in other studies (Ichikawa et al., 1971; Hoa et al., 2012).

\section{Xoo races}

In the present study, a collection of 10 pathogen races of Xoo were tested. These were named R1, R2, R3, R4, R5, R6, R7, R8, R9 and R10 (Ton et al., 2003; Hoa et al., 2012). Of the 10 races, eight were found from many provinces of northern Vietnam, with two (R9 and R10) from Japan. The races were isolated from rice BB-infected leaves that were collected from the main rice-cultivating areas of northern Vietnam. Among the 10 Xoo races, $\mathrm{R} 2$ and $\mathrm{R} 3$ were the most widely distributed.

\section{Media}

In addition to the media [Wakimoto medium (WM), Yeast extract-soluble starch medium(YS), Antibiotic producing medium (APM), Yeast-extract malt medium (YM), Muller Hinton agar (MHA); Ashby medium (AM) and Nutrient Agar (NA)] described in our previous paper (Hoa et al., 2012), we used five other media [Medium 2M, Medium 301, Medium A-16, Medium no. 8, Medium A-3M] in the present study.

\section{Detection of actinomycetes capable of inhibiting Xoo races in vitro}

The antibacterial activity of actinomycetes against 10 pathogenic $X_{0 o}$ races was tested by the agar-piece method $(6 \mathrm{~mm})$ on Wakimoto medium as previously described (Hoa et al., 2012). In brief, the actinomycete isolates were inoculated on YS medium at room temperature $\left(25\right.$ to $\left.30^{\circ} \mathrm{C}\right)$ for 7 to 14 days. The pieces of agar $(6 \mathrm{~mm})$ that contained actively-growing actinomycete isolates were cut and placed onto the test plates culturing races of $X_{0 O}$. The plates were incubated at $28 \pm 1^{\circ} \mathrm{C}$ for two to four days and the inhibitory effect of the actinomycete isolates on Xoo growth was evaluated by measuring inhibition zones $(\mathrm{cm})$. To prepare the test plates, races of Xoo were cultured in Wakimoto broth to a concentration of ca. $10^{8} \mathrm{CFU} / \mathrm{ml}$. 
$150 \mathrm{ml}$ of Wakimoto agar was autoclaved and cooled down to 45 to $50^{\circ} \mathrm{C}$ and then mixed with $10 \mathrm{ml}$ of the Xoo cultures. This mixture was poured into Petri dishes that were used as the test plates (Hoa et al., 2012).

\section{Test for antibiosis to microorganisms}

The actinomycete isolates inhibiting all 10 Xoo races were tested for their antibiosis to four indicator microogarnisms, M. luteus NBRC 13867, E. coli NBRC 14237 , B. subtilis NBRC 3134 and S. cerevisiae NBRC 10217 (indicator microorganisms were provided by NITE, Japan), and to two beneficial bacteria, Azotobacter sp. (VTCC-B-106), which is well-known in nitrogen fixation, and $P$. putida VTCC-B-657 which degrades phosphors into accessible forms for plants. Antibiosis tests were performed by using the agar-piece method as mentioned previously (Ichikawa et al., 1971; Fredrickson and Elliott, 1985; Blackburn and Avery, 2003).

\section{Selection of medium for antibiotic production of the potential actinomycete strain}

Seven media [Antibiotic producing medium (APM), Modified-APM (MAPM), Medium 2M, Medium 301, Medium A-16, Medium no. 8, Medium A-3M] were used for selection of a suitable antibiotic inducing medium of actinomycetes. The method was described previously (Hoa et al., 2012).

\section{Biocontrol of BB in rice by actinomycetes in field trials}

Artificial infection of Xoo races $R 2$ and $R 3$ on rice leaves

The rice cultivars Oryza sativa L. SS1 and Oryza sativa L. KD18 were chosen for field trials for two cropping seasons in 2011 because they are widely cultivated across Vietnam and have susceptibility to the Xoo races. The BB pathogens $\mathrm{R} 2$ and $\mathrm{R} 3$, being the most prevalent in northern Vietnam, were inoculated in Wakimoto medium flasks and incubated at $28^{\circ} \mathrm{C}$ for 48 hours. The Xoo bacterial cells were then diluted in sterilized water to reach a concentration of $10^{8} \mathrm{CFU} / \mathrm{ml}$ to infect the two rice cultivars. This method was described by Ji et al., (2008).

The two rice cultivars were planted in the field. Artificial infection was conducted at the rice booting stage because this stage is the most susceptible to BB and infection during this stage subsequently impacts rice yields. Infection was performed by dipping the scissors into a Xoo bacterial suspension and using them to clip the tip of the rice leaves (Kauffman et al., 1973). After 14 days, the length of lesions on 15 leaves per rice cluster was measured at the field. Three rice clusters per treatment formula were examined.

Xoo treatment by actinomycetes
Actinomycetes were cultured in MAPM broth with shaking at 150 cycles/minute for five days. From this, $250 \mathrm{ml}$ of the cultured solution was used for spraying over an area of $1 \mathrm{~m}^{2}$ in each experimental square.

Treatments

Each of the two rice cultivars (Oryza sativa L. SS1 and Oryza sativa L. KD18) was planted in 45 experimental blocks within an experimental rice field at Hanoi University of Agriculture. The biocontrol trials at the rice field were conducted during two growing seasons in 2011, which consisted of planting in October and harvest in May and another planting in the end of May and harvest in November. Three squares were used for each treatment as follows.

Treatment 1: (Positive control) rice cultivars were not infected by the Xoo races and were not treated with VN08-A-12. This was done to measure the noninfected/non-treated baseline yield of each paddy for each rice cultivar.

Treatment 2: Rice cultivars were not infected by the Xoo races, but they were treated with VN08-A-12. This experiment was conducted to know the effect of VN08A-12 on the rice yield of each rice cultivar.

Treatment 3: Infected rice cultivars were treated as follows: CT1 denotes spraying VN08-A-12 after 1-day infection by Xoo races; CT2 denotes spraying VN08A-12 after 3-day infection by Xoo races; CT3 denotes spraying VN08-A-12 after 7-day infection by Xoo races; and CT4 denotes spraying VN08-A-12 3 times: after 1, 3 and 7 -day infection by $X O O$ races.

Treatment 4: Rice cultivars were infected by $X o 0$ races but were not treated with the VN08-A-12 broth culture. This was done to measure the effect of $X_{0 O}$ races on the host rice cultivars.

Measurement of the yield of a paddy

To measure and estimate paddy yield for each treatment, the rice grain dry weight from three individual rice plants per rice cluster was measured (Ton et al, 2003).

\section{Identification of VNO8-A-12}

Identification of VN08-A-12 was based on morphological, chemical and molecular methods as mentioned by Sakiyama et al. (2009).

\section{Statistical analysis}

Data from laboratory and rice field experiments were analyzed and subjected to analysis of Variance (ANOVA) and correlation (SPSS Sofware). The significance of each treatment through two rice seasons and for each rice cultivar were determined by ANOVA (Tukey's posthoc test) ( $P$ values $\leq 0.05$ ). 
TABLE 2. Ability of 167 actinomycete strains to inhibit tested Xoo races

\begin{tabular}{|c|c|c|c|c|c|c|c|c|c|c|c|}
\hline $\begin{array}{l}\text { Number of Xoo strains } \\
\text { which actinomycete } \\
\text { strains can inhibit }\end{array}$ & 0 & 1 & 2 & 3 & 4 & 5 & 6 & 7 & 8 & 9 & 10 \\
\hline $\begin{array}{l}\text { Percentage of } \\
\text { actinomycete } \\
\text { Strains }\end{array}$ & $17.9 \%$ & $8.4 \%$ & $7.8 \%$ & $5.9 \%$ & $10.2 \%$ & $2.4 \%$ & $8.4 \%$ & $10.8 \%$ & $11.9 \%$ & $5.9 \%$ & $10.2 \%$ \\
\hline
\end{tabular}

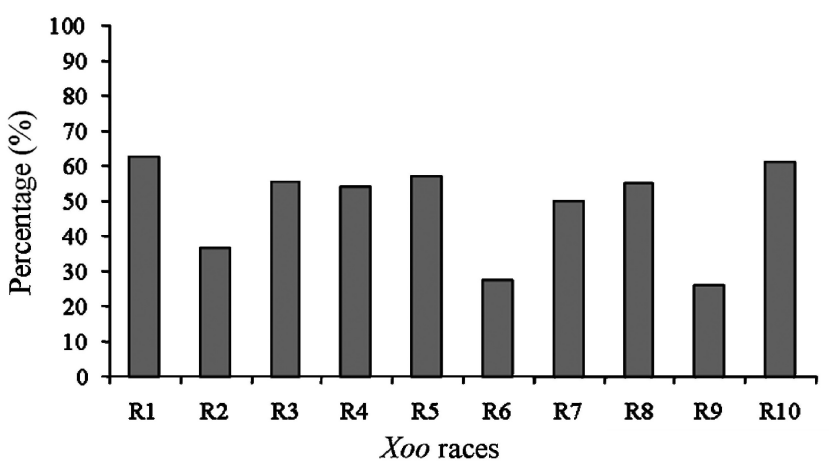

FIG. 1. proportion of the actinomycete strains that inhibits each $\mathrm{Xoo}$ race

\section{RESULTS}

\section{Screening for actinomycete isolates capable of inhibiting $X 00$ races in vitro}

From 2690 actinomycete strains, a total of 167 strains showed high activity against $M$. luteus or $E$. coli and were subsequently chosen for further antagonistic tests against the 10 Xoo races. The inhibition pattern against each $X_{00}$ race showed that races $\mathrm{R} 9, \mathrm{R} 6$ and
R2 expressed the highest levels of inhibition relative to the others (Figure1). Only 25.7, 27.1 and 36.5\% of the actinomycete strains inhibited races R9, R6 and R2, respectively. Inhibition of the other races of actinomycete strains accounted for at least $49.7 \%$. Most of the tested actinomycete isolates (73\%) were able to inhibit more than 2 Xoo races. A total of 17 strains (10.2\%) were found to be able to inhibit all 10 Xoo races (Table 2).

The inhibition zones of the 17 actinomycete strains inhibiting 10 Xoo races varied from 0.2 to $2.6 \mathrm{~cm}$, depending on the strains and $X_{0 O}$ races (data not shown).

\section{Test of antibiosis to microorganisms}

The purpose of this study was to select actinomycete strains capable of inhibiting all 10 Xoo races while not being harmful to other microorganisms. The 17 actinomycete strains capable of inhibiting all 10 Xoo races chosen above were tested for their inhibition activity against four indicator microorganisms (M. luteus, E. coli, B. subtilis and S. cerevisiae) and two useful microorganisms (Azotobacter sp. and P. putida).

TABLE 3. Inhibition activity of the 17 actinomycete strains against tested microorganisms

\begin{tabular}{|c|c|c|c|c|c|c|c|}
\hline \multirow{2}{*}{ No. } & \multirow{2}{*}{ Name } & \multicolumn{6}{|c|}{ Inhibition zones (cm) } \\
\hline & & M. luteus & E. coli & S. cerevisiae & B. subtilis & Azotobacter sp. & P. putida \\
\hline 1 & VN06-A-353 & - & - & $1.4 \pm 0.1$ & - & n.d & n.d \\
\hline 2 & VN06-A-379 & $0.8 \pm 0.1$ & $2.0 \pm 0.2$ & - & + & n.d & n.d \\
\hline 3 & VN06-A-1098 & $1.8 \pm 0.2$ & $1.5 \pm 0.4$ & + & $1.0 \pm 0.2$ & n.d & n.d \\
\hline 4 & VN06-A-1457 & $1.1 \pm 0.1$ & $2.4 \pm 0.2$ & - & + & n.d & n.d \\
\hline 5 & VN06-A-1477 & $0.6 \pm 0.3$ & $1.1 \pm 0.1$ & + & $1.0 \pm 0.1$ & n.d & n.d \\
\hline 6 & VN08-A-12 & $1.6 \pm 0.1$ & - & - & - & - & - \\
\hline 7 & VN08-A-306 & $1.2 \pm 0.1$ & - & - & - & n.d & n.d \\
\hline 8 & VN08-A-352 & $2.3 \pm 0.1$ & - & - & + & n.d & n.d \\
\hline 9 & VN08-A-395 & $1.2 \pm 0.3$ & $0.8 \pm 0.2$ & - & + & n.d & n.d \\
\hline 10 & VTCC-A-99 & + & - & - & - & n.d & n.d \\
\hline 11 & VTCC-A-289 & $1.0 \pm 0.1$ & $0.5 \pm 0.1$ & + & + & n.d & n.d \\
\hline 12 & VTCC-A-363 & + & - & - & $1.8 \pm 0.1$ & n.d & n.d \\
\hline 13 & VTCC-A-367 & $1.2 \pm 0.2$ & $0.5 \pm 0.2$ & - & $1.0 \pm 0.2$ & n.d & n.d \\
\hline 14 & VTCC-A-378 & + & - & $1.0 \pm 0.2$ & $1.0 \pm 0.1$ & n.d & n.d \\
\hline 15 & VTCC-A-456 & + & - & $1.2 \pm 0.1$ & - & n.d & n.d \\
\hline 16 & VTCC-A-465 & $2.3 \pm 0.1$ & - & - & $2.0 \pm 0.3$ & n.d & n.d \\
\hline 17 & VN10-A-44 & - & - & - & $1.4 \pm 0.1$ & - & $1.0 \pm 0.1$ \\
\hline
\end{tabular}

Note: - indicates that the actinomycete strain did not inhibit the indicator microorganism; + indicates that the strain has antagonistic activity, but the inhibition zone was too small to measure. N.d. denotes not done. Each value is an average of a 3 time-repeated assay. 
We found that strains VN06-A-353, VN08-A-12, VN08-A-306, VN08-A-352, VTCC-A-99 and VN10-A-44 only inhibited one of the four indicator microorganisms whereas the others inhibited more (Table 3). It was observed that VN08-A-12 and VN10-A-44 grew faster than the other strains. After three days of cultivation, they showed high inhibition activity against $X_{0 o}$ races while the other strains required from five to seven days of cultivation to express this activity (data not shown). Thus, only two strains VN08-A-12 and VN10-A-44 were checked for their inhibition potential against the two beneficial microorganisms such as Azotobacter sp. (VTCC-B-106) and P. putida VTCC-B-657. The strain VN10-A-44 inhibited P. putida; whereas VN08-A-12 did not inhibit either of the beneficial bacteria tested. Therefore, VN08-A-12 was selected for further analysis and for the rice field trials.

\section{Selection of the best medium for fermentation of VN08-A-12}

Seven media were used to find the best medium for antibiotic production of VN08-A-12. From this, MAPM was found to be the best medium for VN08-A-12 to produce bioactive compounds. The inhibition zones measured when cultured in MAPM were significantly higher than those cultured in the other media (data not shown).

\section{Biological control of Xoo races by VN08-A-12 in the rice field trials}

Xoo lesion length of rice cultivars SS1 and KD18

Inhibition efficacy of VN08-A-12 on rice cultivars was evaluated by $\mathrm{XoO}_{\mathrm{O}}$-induced lesion length and rice yield following two rice growing seasons. The Xoo lesion lengths on rice leaves are shown for two rice cultivars SS1 (Figure 2A) and KD18 (Figure 2B). Bacterial blight disease occurred differently in the two rice cultivars, with SS1 being more severely infected by XoO races than $\mathrm{KD} 18$, as shown in the Xoo lesion lengths that were from 15.2 to $25.5 \mathrm{~cm}$ for SS1 and from 7.2 to $12.9 \mathrm{~cm}$ for KD18. Treatment with VN08-A-12 significantly reduced the Xoo lesion length in rice leaves in both rice seasons. For example, in the R2-infected SS1 cultivar, the treatment after a 1-day infection reduced the Xoo lesion length by $25.3 \%$ and $33.4 \%$ reductions in the spring and summer seasons, respectively; 3-time treatment by VN08-A-12 reached $32.6 \%$ and $38.3 \%$ reductions in the spring and summer seasons, respectively. Lesion lengths in the R3-infected SS1 cultivar were reduced by $27.5 \%$ after a 1 -day infection in both rice seasons and by $15.2 \%$ and $29.4 \%$ after 3-time treatments by VN08-A-12 in the spring and summer seasons, respectively. On the other hand, for the R2-infected KD18 cultivar, the treatment by VN08-

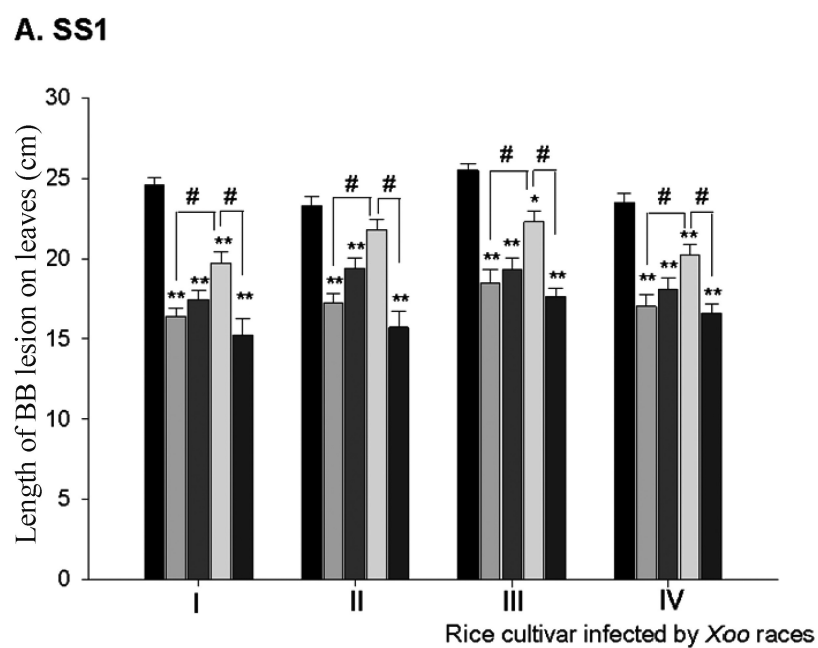

\section{B. KD 18}

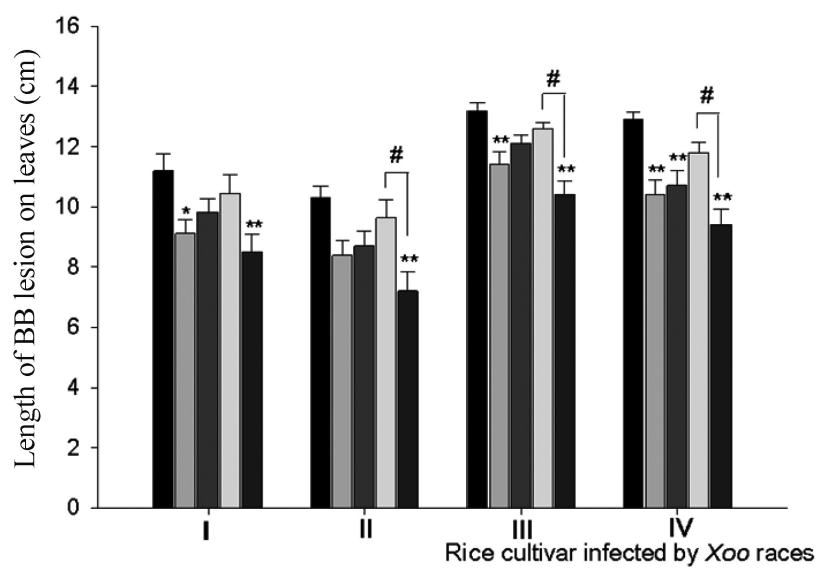

CT0: Without VN08-A12 treatment

$\square$ CT1: With VN08-A12 treatment after a 1-day infection

CT2: With VN08-A12 treatment after a 3-day infection CT3: With VN08-A12 treatment after a 7-day infection

CT4: With 3 times of VN08-A12 treatment after 1, 3 and 7 day infection

FIG. 2. Effect of VN08-A12 on Xoo lesion length on leaves

SS1 (A) and Khang dan (B) in spring and summer rice seasons. The leaves were artificially infected by $X$ oo R2 (R2) or $X$ oo R3 (R3) in summer and spring seasons without VN08-A12 treatment (CTO), with a single treatment of VN08A12 after 1-day infection (CT1), 3-day infection (CT2) and 7-day infection (CT4) or with three-time treatments of VN08A12 after 1, 3 and 7- day infections. I refers to R2 in Summer; II refers to R2 in Spring; III refers to R3 in Summer; IV refers to R3 in Spring. ANOVA was tested following by Tukey's posthoc test. ${ }^{*} p<0.05,{ }^{*} * p<0.005$ indicate significant differences between CT1, CT2, CT3, CT4 to the respective control (CTO); \# $\mathrm{p}<0.05$ indicates significant difference between CT4 and other groups.

A12 after a 1-day infection had lesion lengths reduced by $18 \%$ in both seasons, and $29.8 \%$ and $24.1 \%$ (in the spring), $15.2 \%$ and $29.4 \%$ (in the summer) after the 3-timetreatments by VN08-A-12; R3-infected KD18 cultivar when treated 3 times with VN08-A12 had XoO 
lesion lengths in leaves reduced by $27.2 \%$ and $21.2 \%$ in the spring and summer seasons, respectively. The efficacy of VN08-A12 to suppress Xoo races was less when treatment occurred late. Results showed that the treatment after a seven day infection reduced the lesion length from $6.1 \%$ to $12.6 \%$. This suggests that late treatment by VN08-A-12 resulted in a lower level of inhibition of $X_{0 O}$ races.

\section{Paddy yield of rice cultivars: SS1 and KD18} SS1 cultivar

Results from the SS1 cultivar paddy yield following the spring growing season are shown in Table 4. When infected by Xoo races, the SS1 cultivar paddy yield was reduced by $53.5 \%$ (infected by R2) and $48.4 \%$ (infected by R3). When treated with VN08-A-12, the yield of the paddies of the R2-infected SS1 cultivar increased $24.3 \%-26.8 \%$ (one-time treatment) and $43.2 \%$ (three-time treatment). The yield of the paddies of the R3-infected SS1 cultivars increased $26.8 \%$ $30.5 \%$ (one-time treatment) and $35.7 \%$ (one-time treatment) in comparison with the Xoo-infected SS1 cultivar without VN08-A-12 treatment. Notably, the VN08-A12 treatment increased the yield of the cultivar paddies from $13.16 \%$ (one-time treatment) to 16.98 $\%$ (three-time treatment). Overall, the productivity of SS1 cultivar paddies both healthy and infected with XoO races was improved when treated with VN08-A-12.
In summer season, the same trend of reduced rice yields was observed (data not shown). R2 and R3 Xoo caused greater reductions in rice yield of the SS1 cultivar than those in spring season; however, VN08A-12 also reduced the yield loss of the SS1 cultivar. We found that R2 and R3 races caused more than a $50 \%$ reduction in rice yield of the SS1 cultivar (58.2 $\%$ for R2 and $55.1 \%$ for R3). The yield of the paddies of the R2-infected SS1 cultivar increased $20.7 \%$ $25.5 \%$ (one-time treatment) and $36.6 \%$ (threetime treatment); that of the R3-infected SS1 cultivar increased $20.2 \%-25.7 \%$ (one-time treatment) and $38.5 \%$ (three-time treatment) in comparison with the Xoo-infected SS1 cultivar without VN08-A-12 treatment. It was observed that a three-time treatment with VN08A-12 increased the rice yield by $2.66 \%$ of the healthy SS1 cultivar in comparison with the untreated healthy rice.

KD18 cultivar

Table 5 shows results on the rice yield of KD18 cultivar. Xoo races R2 and R3 significantly reduced the paddy yield of KD18 by $33.31 \%$ for R2 and $44.05 \%$ for R3. Results showed that VN08-A-12 improved the paddy yield of the rice cultivar. In the spring season, treatment with VN08-A-12 increased the rice yield $9.4-29.4 \%$ in the R2-infected KD18 cultivar. On the other hand, the paddy of R3-infected KD18 cultivar treated with VN08-A-12 increased $20.75-38.99 \%$ in

TABLE 4. Effect of VN08-A-12 on the productivity of Oryza sativa L. SS1 in the spring cropping season, 2011

\begin{tabular}{|c|c|c|c|c|c|c|c|c|c|}
\hline \multirow{2}{*}{$\begin{array}{c}\text { Treatment } \\
\text { by VN08-A-12 }\end{array}$} & \multicolumn{3}{|c|}{ Infected by R2 } & \multicolumn{3}{|c|}{ Infected by R3 } & \multicolumn{3}{|c|}{ Without infection } \\
\hline & $\mathrm{G} / \mathrm{INV}$. & ORY $(\%)$ & $\operatorname{RRY}(\%)$ & $\mathrm{G} / \mathrm{INV}$ & ORY $(\%)$ & $\mathrm{RRY}(\%)$ & G/INV. & $\operatorname{ORY}(\%)$ & $\operatorname{IRY}(\%)$ \\
\hline Positive control & $55.05 \pm 0.93(a)$ & 100 & 0.00 & $55.05 \pm 0.93(a)$ & 100 & 0.00 & $55.05 \pm 0.93(\mathrm{a})$ & 100 & \\
\hline Negative control & $25.60 \pm 3.14(b)$ & 46.50 & 53.50 & $28.60 \pm 2.95(b)$ & 51.60 & 48.40 & n.d. & & \\
\hline After a 1-day infection & $40.40 \pm 1.22(c)$ & 73.38 & 26.62 & $45.20 \pm 2.15$ (ef) & 82.10 & 17.90 & $62.30 \pm 0.20(\mathrm{~g})$ & 113.16 & 13.16 \\
\hline After a 3-day infection & $40.00 \pm 2.00(c)$ & 72.66 & 27.34 & $43.60 \pm 0.35$ (ef) & 79.20 & 20.80 & $62.30 \pm 0.10(\mathrm{~g})$ & 113.16 & 13.16 \\
\hline After a 7-day infection & $39.00 \pm 1.00(\mathrm{c})$ & 70.84 & 29.16 & $43.20 \pm 1.71(\mathrm{e})$ & 78.47 & 21.53 & $62.30 \pm 0.30(\mathrm{~g})$ & 113.16 & 13.16 \\
\hline At three times treated & $49.40 \pm 1.00(d)$ & 89.73 & 10.27 & $48.10 \pm 1.15(\mathrm{df})$ & 87.37 & 12.63 & $64.40 \pm 0.40(h)$ & 116.98 & 16.98 \\
\hline
\end{tabular}

Notes: G/INVD: gram per rice individual; ORY: obtained rice yields; RRY: Reduced rice yields; IRY: Increased rice yields; n.d., not done Statistical analysis was done by ANOVA following by Tukey's test for each column and each row; groups sharing at least one common letter were not significantly different $(p>0.05)$; groups sharing no common letter were significantly different $(p<0.05)$

TABLE 5. Effect of VN08-A-12 on the productivity of Oryza sativa L. KD 18 in the spring cropping season, 2011

\begin{tabular}{|c|c|c|c|c|c|c|c|c|c|}
\hline \multirow{2}{*}{$\begin{array}{c}\text { Treatment } \\
\text { by VN08-A-12 }\end{array}$} & \multicolumn{3}{|c|}{ Infected by $\mathrm{R} 2$} & \multicolumn{3}{|c|}{ Infected by R3 } & \multicolumn{3}{|c|}{ Without infection } \\
\hline & G/INV. & $\mathrm{ORY}(\%)$ & $\mathrm{RRY}(\%)$ & G/INV. & $\mathrm{ORY}(\%)$ & $\mathrm{RRY}(\%)$ & G/INV. & ORY (\%) & $\mathrm{IRY}(\%)$ \\
\hline Positive control & $63.62 \pm 0.75(a)$ & 100 & 0.00 & $63.62 \pm 0.75(a)$ & 100 & 0.00 & $63.62 \pm 0.75(a)$ & 100 & \\
\hline Negative control & $42.60 \pm 1.51(b)$ & 66.96 & 33.31 & $35.60 \pm 0.72(\mathrm{~g})$ & 55.95 & 44.05 & n.d. & & \\
\hline After a 1-day infection & $61.20 \pm 0.26$ (af) & 96.19 & 3.81 & $58.80 \pm 1.05(h)$ & 92.42 & 7.58 & $65.60 \pm 0.31(1)$ & 103.11 & 3.11 \\
\hline After a 3-day infection & $51.30 \pm 0.30(d)$ & 80.63 & 19.37 & $52.20 \pm 1.15(\mathrm{i})$ & 82.04 & 17.96 & $65.60 \pm 0.10(\mathrm{I})$ & 103.11 & 3.11 \\
\hline After a 7-day infection & $48.40 \pm 1.35(e)$ & 76.07 & 23.93 & $44.80 \pm 1.47(k)$ & 76.70 & 23.30 & $65.60 \pm 0.20(\mathrm{I})$ & 103.11 & 3.11 \\
\hline At three times treated & $60.40 \pm 0.40(f)$ & 94.93 & 5.06 & $60.40 \pm 0.69$ (fh) & 94.93 & 5.06 & $67.40 \pm 0.20(\mathrm{~m})$ & 105.94 & 5.94 \\
\hline
\end{tabular}

Notes: G/INVD: gram per rice individual; ORY: obtained rice yields; RRY: Reduced rice yields; IRY: Increased rice yields; n.d., not done Statistical analysis was done by ANOVA following by Tukey's test for each column and each row; groups sharing at least one common letter were not significantly different ( $p>0.05)$; groups sharing no common letter were significantly different $(p<0.05)$ 
comparison to the Xoo-infected KD18 cultivar without VN08-A-12 treatment. For the healthy KD18 cultivar, a one-time treatment with VN08-A-12 improved the rice paddy $3.11 \%$ while a three-time treatment increased the paddy by $5.94 \%$. The data on the KD18 paddy in the summer season was not obtained, due to rodent damage to the crop.

\section{Identification of VN08-A-12}

Based on the results of morphological and physiological characterization of VN08-A-12, we found that the VN08-A-12 strain had typical characteristics of the Streptomyces genus (data not shown). Analysis of the whole-cell hydrolysate of strain VN08-A-12 showed the presence of a chemotype I cell wall characterized by LLA $A_{2} p m$. No diagnostic sugars were found. The main components of fatty acids were anteios(anteios- $\mathrm{C}_{15}: 0$ ), iso- (iso- $\mathrm{C}_{16}: 0$ ) and normal $\left(n-\mathrm{C}_{16}: 0\right.$ ) acids. The menaquinones were MK-9 $\left(\mathrm{H}_{6}\right) 51.9 \%$ and MK-9 $\left(\mathrm{H}_{8}\right) 48.1 \%$. The GC content was $75 \%$. The cultural and physiological characterization and the DNA homology suggested strain VN08-A-12 is identical to Streptomyces toxytricini.

\section{DISCUSSION}

Bacterial blight disease in rice is one of the major diseases in the rice culture of northern Vietnam as well as other rice-growing regions of the world. Many XoO races have been identified from many rice cultivation areas of Vietnam (Ton et al., 2003). In this study, actinomycete strains that were able to inhibit all major Xoo isolates were screened from a collection of 2960 actinomycete strains. Results showed that actinomycete strains were able to inhibit $X o 0$ races (Figure 1, Table 2) and in particular, 17 strains could inhibit all 10 major Xoo races. Their inhibition assays on indicator microorganisms M. luteus NBRC 13867, E. coli NBRC 14237, B. subtilis NBRC 3134, S. cerevisiae NBRC 10217 and useful bacteria Azotobacter sp. (VTCC-B-106) and $P$. puttida VTCC-B-657 led to our selection of only one strain, namely VN08-A-12, for further rice field study for its potential benefits to the environment.

In this study, for cultivation of VN08-A-12 we chose the suitable medium of MAPM in which soybean meal of the original medium (APM) was replaced with tofu waste. Our previous research found that tofu waste could be used instead of soybean meal for the fermentation of the Streptomyces virginiae strain VN10-A-44 (Hoa et al., 2012). This medium was good for largevolume fermentation of Streptomycete for large-scale applications in agriculture. Our study thus has the potential to recycle tofu waste which is one of the major sources of waste in the production of tofu in Vietnam and other Asian countries.

Our finding that the Streptomyces toxytricini VN08A-12 was able to strongly inhibit all 10 Xoo races while not being harmful to other tested microorganisms is in agreement with a previous study (Wu and Chen, 1995). They found that Streptomyces toxytricini, isolated from soil in Taiwan was capable of producing antibiotics, but appeared to be non-toxic to mice and tomato seedlings. In this study, the Streptomyces toxytricini VN08-A-12 was isolated from dead leaves collected in Vietnam by using a dry heating method in 2008 (Hop et al., 2011). The leaf source of VN08-A-12 isolation suggests that the Streptomyces toxytricini VN08A-12 may be easily adapted to the rice field both on rice leaves and in the soil. To our knowledge, this is the first report of Streptomyces toxytricini being a potential biological agent for controlling BB in rice whereas Lysobacter antibiocus isolated from the rhizosphere of rice in China (Ji, et al., 2008) and Streptomyces bottropensis isolated from soil in Korea (Park et al., 2011) were recently reported.

The rice field trials for the two rice cultivars and two rice cropping seasons were evaluated based on lesion lengths and rice yields. In general, the Streptomyces toxytricini VN08-A-12 was able to significantly suppress the $X_{O O}$ races in natural rice fields. These results were in agreement with a previous study by Hastuti et al. (2012), who showed that Streptomyces spp. may be a potential candidate for biologically controlling BB in rice. On the other hand, Streptomyces toxytricini VN08-A-12 was shown to significantly reduce the yield loss of $X_{0 O}$ infected rice cultivars in the present study.

For the SS1 cultivar, which is often heavily affected by $\mathrm{BB}$, the lesion lengths varied from $23.3 \mathrm{~cm}$ to $25.5 \mathrm{~cm}$ in the two seasons. VN08-A-12 significantly reduced Xoo lesion lengths (Figure 2). In the spring cropping season, late treatment by VN08-A-12 in the rice cultivar infected by Xoo R2 (after a seven day infection) reduced Xoo lesion lengths by $6.1 \%$, whereas early and multiple treatments brought reductions of greater than $32 \%$. Xoo lesion lengths were shorter when SS1 was infected by R3 compared to R2, and this was similarly recorded in the summer cropping season (Figure 1).

For the KD18 cultivar, Xoo lesion lengths were less reduced (29.8\% reduction for R2-infected and 27.2 \% R3-infected crops in the spring season; $24.1 \%$ and $21.2 \%$ for crops infected by R2 and R3 in the summer season, respectively). The effects of Xoo reduction in the two tested rice cultivars in the spring season were more pronounced than in the summer season. This could have been due to the fact that the summertime climate favors BB and that summer heavy rains could have affected the treatment with VN08-A-12 in the rice 
field. In Vietnam, BB is more severe and causes highest losses in the summer copping season (Ton et al, 2003; E et al., 1999). Figure 2 shows the same trend that earlier and multiple treatments with VN08-A12 leads to effective control of BB in the SS1 and KD18 cultivars.

Results from measured rice yields of the tested cultivars showed that the Streptomyces toxytricini VN08$\mathrm{A}-12$ reduced $\mathrm{BB}$ caused by races $\mathrm{R} 2$ and $\mathrm{R} 3$ and this corresponded to higher rice yields. This positive effect depended on the rice cultivar, Xoo races, the rice copping season and the timing of the treatment with VN08-A-12. For example, the paddy of cultivar SS1 infected by race R2 had a rice yield ranging from 70.8 to $73.8 \%$, with a one-time treatment with VNO8A-12 in the spring season (at 1, 3 and 7 day infection); however these were higher than those infected by Race R2 that were not treated with VN08-A-12, which showed $46.5 \%$ paddy yields (reduced $53.5 \%$ ). In the case of cultivar SS1 infected by race R3, its yields from paddies were from 78.4 to $82.1 \%$ with a onetime treatment and $51.6 \%$ (48.4\% reduction) without treatment by VN08-A-12. In particular, a three-time treatment with VN08-A12 (with $87.37 \%$ paddy yield) significantly improved the rice yield of cultivar SS1. This was also observed in the summer season (data not shown). Interestingly, it was found that the rice yield of the healthy cultivar SS1 treated with VN08-A12 was significantly higher than that of the untreated one and these results were observed for all the treatments in both the spring and summer seasons. This may be a result of hormesis. It is common in animal/plant physiology that the bacteria improvies the physiology of the plant, turns on genes, or produces hormones (Jocelyn, 2003).

For cultivar KD18, treatment by VN08-A-12 significantly reduced the effect of Xoo infection (Table 5). KD18 yields increased from 9.4 to $38.9 \%$ following the treatment by VN08-A-12. In particular, treatments by VN08-A-12 in healthy KD18 showed significantly increased rice yields from 13.6 to $16.9 \%$. These results suggest that this particular strain could be used as a rice fertilizer. Previous studies showed that plantpromoting rhizobacteria induce systemic resistance in rice against BB (Chithrashree et al., 2011). The improvement of cultivar paddies by treatment with VN08-A-12 could be possibly due to the bioactive compounds that inhibit $X_{O O}$ and to the nutrient contents of the VN08-A-12 fermented culture. A previous study (Hastuti et al., 2012) also found that Streptomyces isolates increased plant yield in comparison to the chemically-sprayed and non-treated plots. Our results suggested that VN08-A-12 has potential application for the biological control of $\mathrm{BB}$ and for the improvement of rice yields. Further examination on large-scale trials of rice fields should be performed in the future.

\section{ACKNOWLEDGEMENTS}

This research was funded by the Vietnam National Foundation for Science and Technology Development (NAFOSTED) under grant number 106.03-2010.34. We would like to thank the leaders of a joint research project between the Institute of Microbiology and Biotechnology, Vietnam National University Hanoi, Vietnam (VNUH-IMBT) and the Biological Resource Center, National Institute of Technology and Evaluation (NBRC), Japan for providing the microorganisms in this study. We would like to thank Dr. Todd W. Miller, Division of Fish and Wildlife, Marianas Protectorate Saipan (USA) for his critical review of this paper. We would like to thank the Korean Collection for Type Cultures (KCTC), Biological Resource Center, Korea Research Institute of Bioscience and Biotechnology, Korea (KRIBB) for organizing an international training course on microbial taxonomy, which provided technical and material support to identify VN08-A-12 to the species level. We also thank Dr. Hiroshi Kinoshita, the International Center for Biotechnology, Osaka University, for his technical support at VNUH-IMBT.

\section{REFERENCES}

Adhikari, T.B., Mew, T.W., and Teng, P.S. (1994) Progress of bacterial blight on rice cultivars carrying different $X a$ genes for resistance in the field. Plant Dis., 78, 73-77.

Anzai, K., Nakashima, T., Kuwahara, T., Suzuki, R., Ohfuku, Y., Takeshita, S., and Ando, K. (2008) Actinomycete bacteria isolated from the sediments at coastal and offshore area of Nagasaki prefecture, Japan: diversity and biological activity. J. Biosci. Bioeng. 2, 215-217.

Blackburn, A.S., and Avery, S.V. (2003) Genome-wide screening of Saccharomyces cerevisiae to identify genes required for antibiotic insusceptibility of eukaryotes. Antimicrob. Agents and Chemother., 47, 676-681.

Chithrashree, A., Udayashankar, C., Chandra, N.S., Reddy, M.S., and Srinivas, C. (2011) Plant growth-promoting rhizobacteria mediate induced systemic resistance in rice against bacterial leaf blight caused by Xanthomonas oryzae pv. oryzae. Biol. Control., 59, 114-122.

Dinh, H.D., Oanh, N.K., Toan, N.D., Du, P.V., and Loan, L.C. (2008) Pathotype profile of Xanthomonas oryzea pv oryzea isolated from the rice ecosystem in Cuulong river delta. Omorice, 16, 34-40.

E, L.V., Noda, T., and Du, P.V. (1999) Resistance assessment of rice cultivars to Xanthomonas oryzea pv. oryzea and pathogenicity testing of bacterial leaf blight isolates in Vietnam. Omorice, 7, 120-130.

Fredrickson, J.K., and Elliott, L.F. (1985) Effects on winter wheat seedling growth by toxin-producing rhizobacteria. Plant and Soil, 83, 399-409.

Gnanamanickam, S. S. (2009) Biological control of bacterial blight of rice. In Biological control of rice diseases. Springer, pp: 67-78. $108 \mathrm{p}$.

Hastuti, R.D., Lestari, Y., Saraswati, R., Suwanto, A., and 
Chaerani. (2012) Capability of Streptomyces spp. in controlling bacterial leaf blight disease in rice plants. Am. J. Agr. Biol Sci., 7 (2), 217-223.

Hoa, P.T.P., Quang, N.D., Sakiyama, Y., Hop, D.V., Hang, D.T., Van, N.T., Quy, N.T.K., and Dao, N.T.A. (2012) Screening for actinomycetes isolated from soil able to inhibit Xanthomonas oryzae pv. oryzae causing rice bacterial blight disease in Vietnam. Afr. J Biotechnol., 11 (80), 14586-14594.

Hop, D.V., Sakiyama, Y., Binh, C.T.T., Otoguto, M., Hang, D.T., Miyadoh, S., Luong, D.T., and Ando, K. (2011) Taxonomic and ecological studies of actinomycetes from Vietnam: isolation and genus- level diversity. J. Antibiot (Tokyo), 1-8.

Ichikawa, T., Date, M., Ishikura, T., and Ozaki, A. (1971) Improvement of kasugamycin-producing strain by the agar piece method and the prototroph method. Folia Microbial., 16, 218-224.

Ji, G.H., Wei, L.F., Wu, Y.P., and Bai, X.H. (2008) Biological control of rice bacterial blight by Lysobacter antibioticus strain 13-1. Biol. Control., 45, 288-296.

Jocelyn, K. (2003) Hormesis: Sipping from a poisoned chalice. Science, 302, 376-379.

Kauffman, H.E., Reddy, A.P.K., Hsieh, S.P.Y., and Merca, S.D. (1973) An improved technique for evaluating resistance of rice varieties to Xanthomonas oryzea. Plant Dis. Report., 57, 537-541.

Ndonde, M.J.M., and Semu, E. (2000) Preliminary characterization of some Streptomyces species from four Tanzanian soils and their antimicrobial potential against selected plant and animal pathogenic bacteria. World J. Microb. Biotechnol., 16, 595-599.

Okami Y., and Hotta K (1988) Search and discovery of new antibiotics, In: Goodfellow M, Williams ST, Mordarski M (ed). Actinomycetes in Biotechnology. Academic Press, Inc., San Diego, pp. 33-67.

Park, S.B., Lee, I.A., Joo-Won, S., Jeong-Gu, K., and Lee, H.W. (2011) Screening and identification of antimicrobial compounds from Streptomyces bottropensis suppressing rice bacterial blight. J. Microbiol. Biotechnol., 21, 1236-1242.

Sakiyama, Y., Thao, N.K.N., Giang, N.M., Miyadoh, S., Hop, D.V., and Ando, K. (2009) Kineosporia babensis sp. nov., isolated from plant litter in Vietnam. Internation J. System. Evolut. Microbiol., 59, 550-554.

Ton, P. H., Thuy, B. T., and Hai, T. V. (2003) Pathogenicity of the Bacterial Leaf Blight Strains from Northern Vietnam. The $2^{\text {nd }}$ National Conference on Plant Pathology and Molecular Biology (In Vietnamese).

Wu, R.Y., and Chen, M.H. (1995) Identification of the Streptomyces strain KS3-5. Bot. Bull. Acad. Sin., 36, 201-205.

Yan, M. V., Da, Q. T., Shi, M. T., and Ding, Z. (2000) The antagonism of 26 strains of Streptomycetes sp. against several vegetables pathogens. Hebei Agr. Univer., 23, 65-68. 